www.jmscr.igmpublication.org

Index Copernicus Value: 79.54

ISSN (e)-2347-176x ISSN (p) 2455-0450

crossrefDOI: https://dx.doi.org/10.18535/jmscr/v7i2.71

\title{
A rare case of acute lymphoblastic leukemia/lymphoma with spill over in inguinal lymph node in a middle aged patient
}

\author{
Authors \\ Swati Sharma, Nidhi Varshney, Shyam Kumar Maurya \\ VPIMS \\ Email:pavaninitin85@gmail.com
}

\section{Introduction}

Acute lymphoblastic leukemia (ALL) is a cancer of lymphoid blood cells characterized by large numbers of immature lymphocytes. ALL progresses rapidly and is fatal if left untreated. ${ }^{(1)}$ Precursor lymphoid neoplasms are categorized as $\mathrm{B}$ and $\mathrm{T}$ lymphoblastic leukemia/lymphoma. ${ }^{(2)}$ Leukemia and lymphoma are overlapping clinical presentations of the same disease (i.e, BALL/LBL); diagnosis and classification do not distinguish between these entities. B-cell acute lymphoproliferative disease manifest as pure leukemia (B-ALL) in $80 \%$ cases, isolated extra medullary disease (B-LBL) in $10 \%$ and mixed BALL/B-LBL in $10 \%$ cases. $^{(3)}$ B-ALL/B-LBL is rare in adults and cases with spill-over in inguinal lymph nodes has not been reported as per our knowledge.

\section{Case History}

A 52 year old male reported with the chief complaints of pain abdomen, dyspepsia, pain in shoulder and generalized weakness for 5 months. On physical examination, patient had pallor and enlarged bilateral inguinal lymph nodes. On systemic examination patient had enlarged liver and spleen rest of the systems were within normal limits. Ultrasonography revealed hepatosplenomegaly.

In hematological investigations, patient's total leucocyte count was normal with low platelet count of 32000/cumm. Peripheral smear showed RBCs with moderate anisopoikilocytosis with evidence of $0-1$ nucleated RBCs/100 WBCs. Atypical lymphocytes of blastoid morphology were seen. Rests of the hematological and routine serological investigations, urine routine and microscopic examination were within normal limits. Chest X-Ray was normal.

Fine needle aspiration (FNA) from inguinal lymph node was performed. Smears were prepared and stained by MGG and H:E technique. On microscopic examination, smears were cellular consisting of predominantly medium to large lymphoid cells with enlarged nuclei and scanty, finely granular and basophilic cytoplasm admixed with few mature lymphocytes (Figure.1a,b,c) altogether suggestive of leukemia/lymphoma. 


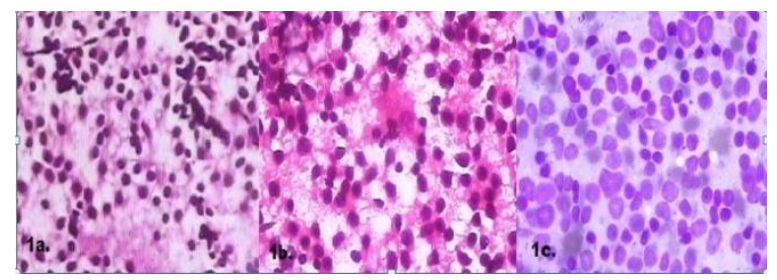

Figure 1 (a,b,c). Fine needle aspiration cytology showing atypical medium to large lymphoid cells (H\&E. \& M.G.G.10x \&40x).

As patient had severe anemia, a bone marrow aspiration was done which revealed a cellular marrow with myeloid, erythroid and megakaryocytic lineage markedly suppressed and replaced by proliferating moderate to large size blast cells comprising $90 \%$ of bone marrow cell population. Blast cells had pleomorphic nuclei with 1-2 nucleoli, clumped chromatin, irregular nuclear membrane and scanty to moderate amount of basophilic cytoplasm (Figure. 2a, b). The impression of bone marrow aspiration examination was ALL.

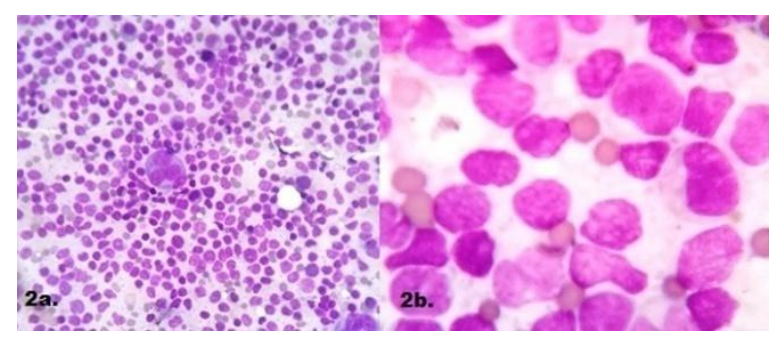

Figure 2(a,b). Bone marrow aspiration showing proliferating moderate to large size lymphoblasts (MGG 40x\&100x).

The patient's blood sample was taken for flow cytometric examination and the results were as follows:

Cell preparation method: Stain-Lyse-Wash

Population gated: Blasts

Gated cells ( $\%$ of all cells): CD45/SSC gating shows $8.5 \%$ blast with negative to dim CD45 and low side scatter which exhibit immunophenotype as shown in Table 1.

Interpretation: The immunophenotypic analysis of gated population of blast $(8.5 \%)$ suggests BLymphoid cell (Precursor B cell) immunophenotype with aberrant expression of CD 13.

B-cell ALL translocation panel was applied on patient's blood sample by Multiplex PCR \& gel electrophoresis technique. The hybrid transcripts for BCR/ABL-1, ETV6/RUNX1, E2A/PBX1 and
MLL/AF4 were not detected in the leukocytes of the sample.

Table 1.

\begin{tabular}{|l|c|c|}
\hline Markers & $\begin{array}{c}\text { Percentage } \\
\text { Positivity }\end{array}$ & Remarks \\
\hline \multicolumn{2}{|l|}{ Non lineage specific } \\
\hline CD 34 & 69.4 & Positive, Moderate \\
\hline Tdt & 27.0 & Positive, DIM \\
\hline HLA -DR & $\mathbf{7 5 . 5}$ & Positive, Moderate \\
\hline \multicolumn{2}{|l|}{ Lymphoid T Cell } \\
\hline CD 3 & 3.7 & Negative \\
\hline CD 4 & 14.1 & Negative \\
\hline CD 5 & 0.5 & Negative \\
\hline CD 7 & 2.4 & Negative \\
\hline CD 8 & 4.8 & Negative \\
\hline CCD 3 & 2.7 & Negative \\
\hline Lymphoid B Cell & \\
\hline CD 10 & 77.8 & Positive, Moderate \\
& \multicolumn{3}{|c|}{ To Bright } \\
\hline CD 19 & 68.0 & Positive, Moderate \\
\hline CD 20 & 5.9 & Negative \\
\hline cCD79a & 20.5 & Positive, Dim \\
\hline CD 13 & 49.5 & Positive, Dim \\
\hline CD 33 & 0.3 & Negative \\
\hline CD 117 & 0.5 & Negative \\
\hline cMPO & 8.6 & Negative \\
\hline CD 11c & 2.4 & Negative \\
\hline
\end{tabular}

\section{Discussion}

ALL is a clonal hematologic disorder. It involves excessive proliferation and impaired differentiation of leukemic blasts that lead to inadequate normal hematopoiesis. Thus, patients usually present with symptoms resulting from bone marrow failure. The extra-medullary form of this disease is rarely reported. However, when found, it most commonly involves the bones, followed by soft tissue, skin and lymph nodes. ${ }^{(4)}$

The WHO classification of tumors of hematopoietic and lymphoid tissues divides B-cell malignancies into precursor B-cell neoplasms and mature B-cell neoplasms. While the rate of remission is high in both adults and children, the 5 year survival rate is much lower in adults. ${ }^{(2)} \mathrm{B}$ ALL/LBL accounts for $2 \%$ of lymphoid neoplasms diagnosed in the United States. ${ }^{(3)}$ Adulthood ALL is a rare disease. In contrast to childhood ALL, survival for adults with ALL is poor. ${ }^{(5)}$ In the present case the patient was a middle aged man. 
On physical examination, peripheral lymph node involvement is seen in $60-80 \%$ of patients. ${ }^{(6)}$ Hepatosplenomegaly is a common finding in patients of ALL and causes abdominal discomfort as seen in our patient.

Baljevic et al stated ALL patients demonstrate anemia and thrombocytopenia to varying degrees. WBC counts at initial presentation can vary significantly, circulating lymphoblast cells are seen on peripheral blood smears in the majority of cases. ${ }^{(7)}$ Similar findings were noted in our case. In leukemia, infiltration of lymph nodes by leukemia cells can occur at any stage of the disease, i.e. during the course of leukemia, prior to onset of marrow leukemia or during relapse. Singhal et al reported a similar case with FNA findings of predominantly immature lymphoid cells conforming to morphology of lymphoblasts with few mature lymphocytes and numerous lymphoglandular bodies in the background as seen in our case. ${ }^{(8)}$ Accurate cytologic diagnosis of extramedullary leukemic infiltration relies on detailed morphologic assessment, clinical examination and relevant laboratory findings. ${ }^{(8)}$

Bone marrow aspiration and biopsy are the definitive diagnostic tests to confirm the diagnosis of leukemia. The diagnosis of ALL is made when at least 30\% lymphoblasts (FAB classification) or $20 \%$ lymphoblasts (WHO classification) are present. ${ }^{(9)}$ In our case BMA revealed more than 90\% blasts.

The most common rearrangement in B-ALL is the $\mathrm{t}(12 ; 21)$ (p13;q22) rearrangement that encodes ETV6-RUNX1 followed by_MLL rearrangements $\mathrm{t}(4 ; 11)(\mathrm{q} 21 ; \mathrm{q} 23) \quad / M L L-A F F 1(A F 4), B C R-A B L 1$ $\mathrm{t}(9 ; 22)(\mathrm{q} 34 ; \mathrm{q} 11) \quad$ and E2A-PBX1 $\mathrm{t}(1 ; 19)$ (q23;p13). ${ }^{(10)}$ But these translocations were not detected in our patient.

In B-lineage ALL the most important markers for diagnosis are CD19, CD20, CD22, CD24, and CD79a, the earliest being CD19, CD22 and CD79a ${ }^{(11)}$ A positive reaction for any two of these three markers, identifies pro-B ALL. ${ }^{(12)}$ In our case, tumor cells were positive for CD34, HLA-
DR, TdT, CD10, CD19, CD79a and CD13 with aberrant expression of CD13.

Our case of precursor B-ALL with spill-over in inguinal lymph node is a rare finding in a middle aged patient. This case further illustrates the need for comprehensive immunophenotyping and genetic testing when establishing an accurate diagnosis of ALL, as this could impact management and will further add to the current knowledge. Prognosis is poor in adults due to high-risk disease characteristics and significant toxicity associated with chemotherapy in adults.

\section{References}

1. Childhood Acute Lymphoblastic Leukemia Treatment. National Cancer Institute. 2017.

2. Hirzel AC, Cotrell A, Gasparini R, Sriganeshan V. Precursor B-Cell Acute Lymphoblastic Leukemia/Lymphoma with L3 Morphology, Philadelphia Chromosome, MYC Gene Translocation, and Coexpression of TdT and Surface Light Chains: A Case Report. Case reports in pathology 2013;2013:679892.

3. Geethakumari PR, Hoffmann MS, Pemmaraju N, Hu S., Jorgensen JL., O'Brien S, Daver, N. Extramedullary B lymphoblastic leukemia/lymphoma (BALL/B-LBL): a diagnostic challenge. Clinical lymphoma, myeloma \& leukemia 2014;14(4):115-118.

4. Siddique MN, Popalzai M, Aoun N et al. Precursor B-cell acute lymphoblastic leukemia presenting as obstructive jaundice: a case report. Journal of Medical Case Reports 2011; 5:269.

5. Pulte D, Jansen L, Gondos A, Katalinic A, Barnes B, Ressing M, et al. Survival of Adults with Acute Lymphoblastic Leukemia in Germany and the United States. Plos one 2014; 9(1):e85554.

6. Tuscano JM, Wang SE, Wun $\mathrm{T}$ et al. Lymphoblastic lymphoma. Drugs and diseases. Medscape. 2015. 
7. Baljevic, Muhamed; Jabbour, Elias; O'Brien, Susan; Kantarjian, Hagop M. Acute Lymphoblastic Leukemia. The MD Anderson Manual of Medical Oncology. 2017; $3^{\text {rd }}$ edition.

8. Singhal N, Tahlan A, Bansal C, Handa U, D' Cruz S. Coexistence of leukemic infiltration and extramedullary hematopoeisis in a lymph node: A cytological diagnosis. Journal of cytology 2011; 28(3): 138-140.

9. Seiter K, Talavera F, Sacher RA, Besa EC. Acute Lymphoblastic Leukemia (ALL) Workup. Medscape;2018.

10. Charles G. Mullighan. Molecular genetics of B-precursor acute lymphoblastic leukemia. Journal of Clinical Investigation 2012;122(10): 3407-3415.

11. Coustan SE, Behm FG, Sanchez J et al. Immunological detection of minimal residual disease in children with acute lymphoblastic leukaemia. Lancet. 1998; 351:550-554.

12. Chiaretti S, Zini G, Bassan R. Diagnosis and Subclassification of Acute Lymphoblastic Leukemia. Mediterranean Journal Of Hematology And Infectious Diseases.2014; 6(1). 